\title{
The Research on the Network Public Opinion Risk Assessment based on the CWAHP-Entropy Method
}

\author{
Chai Wenlei ${ }^{1}$ and Cheng $\mathrm{Mao}^{2}$ \\ ${ }^{1}$ No.180 Wusidong Road. Baoding, Hebei Province, P.R.C \\ ${ }^{2}$ No.289 Lingyusi Street. Baoding, Hebei Province, P.R.C \\ ${ }^{1}$ cwl@hbu.cn,2chengmao82@163.com
}

\begin{abstract}
Since the Internet enters in China, the phenomenon of network public opinion has received the wide range of the attention. The network public opinion has also played the important role in the formation and the spread of the social opinion. At the same time, due to the special nature of the network public opinion, this phenomenon brings the impact on the management mode of the traditional network public opinion. Then, it also leads to the public social events and the false information. Therefore, it is necessary to evaluate the network public opinion. It makes the relevant departments adopt appropriate measures to reduce the risk of the network public opinion. In this paper, we consider to use the combination weight. At the same time, we propose an improved AHP-Entropy method. Aiming at the characteristics of the network public opinion, the method not only considers the subjective weight, but also the objective weight. Then, we get the comprehensive weight. Finally, we use the method to evaluate the risk of the network public opinion. The evaluation results show that the method is reliable and validity.
\end{abstract}

Keywords: Network public opinion; Risk Assessment; Combination weight

\section{Introduction}

The network media has the characteristics of timeliness, interactivity and openness. It makes the network public opinion is different from the traditional media public opinion in the emergence, formation and development [1-2]. The direct, arbitrary and disguised characteristics of the network public opinion led to the social public events, the false information and the bad information [3-4]. Evaluating the risk of the network public opinion and adopting the effective measures to guide, dispose and manage the public opinion events are the new method to resolve effectively the network public opinion [5].

At present, there are few studies on the risk evaluation of the network public opinion at home and abroad. In foreign countries, the research on the network public opinion is closely related to the political and the social connections. Juliane Urban and Kristin Bulkow presented a new approach to measure public opinion on the Internet. With the help of social network analysis and quantitative content analysis public opinion building processes on the Web regarding the Nobel Peace Prize nomination of Barack Obama in 2009 would be examined. And they discussed the strengths and weaknesses of the new approach [6]. Ning Ma and Yi Junliu proposed a Super edge Rank algorithm for opinion leader identification based on super network theory which combined the network topology analysis and text mining. They firstly established a super network model with multidimensional subnetworks. At last, they applied Super edge Rank algorithm to rank super edges, and used the ranking result to identify opinion leaders in opinion super network model [7]. Christopher G. Reddick, Akemi Takeoka Chatfield and Patricia A. Jaramillo examined public opinion on National Security Agency (NSA) mass surveillance programs of Americans. And there were new theory, developed and tested in this paper to explicate the effect of political efficacy on creating greater citizen-centric e-governance. 
The critical discourse analysis of tweets exchanged among citizens and interest groups in Twitter sphere and logistic regression analysis of survey data collected from a random sample public opinion poll of Americans showed the that citizens held strong views toward NSA surveillance programs [8]. Pablo Porten-Cheé and Christiane Eilders tested the spiral of silence theory under conditions of online communication. This study tested the effects of selective exposure to information regarding climate change in Germany. The findings showed no support for the spiral of silence theory. Individuals who saw themselves in the minority were even more likely to express their opinions [9]. Kyungmo Kim, Young Min Baek and Narae Kim examined how news/opinions diffused and how they interacted and influenced each other's ideological frame within agencies sharing the same ideology and between agencies whose ideologies contrasted [10]. In addition, there were many scholars studying the network public opinion [11-13].

In China, Yang Yuanyuan visited the network public opinion that the websites spread. Combining with the characteristics of the network spread, she unscrambled the typical case of network public opinion spread, analyzed the formation and the characteristics of the network public opinion, summarized the supervision and guidance the strategies of the network public opinion. Finally, the author pointed out the problem that the basic study of the network public opinion existed [14]. Liu Hao integrated the journalism, media, communication, social psychology and the computer science to review systematically the characteristics and the formation mechanism of the network public opinion. From the perspective of the network public opinion, he analyzed the typical phenomenon in the network public opinion, explored the formation mechanism of the network public opinion and found the regulatory mechanism of the network public opinion from the origin [15]. Aiming at the shortcomings and the weaknesses of China, Zhou Wei analyzed the problem more systematically and proposed the guidance method of the network public opinion. He made the first case of the violence of the network public opinion as the breakthrough points and expounded the formation of the network public opinion according to analyzing the typical case. In addition, he analyzed the positive effect and the negative effect that the network public opinion brought [16]. Zhong Ying and $\mathrm{Yu}$ Xiucai analyzed 160 major network public opinion events from 1998 to 2009. They found that Chinese network public opinion events developments were rising like a wave. The degree of the network public opinion was closely related to the degree of the regional development. According to revealing the propagation rules of the network public opinion, this paper tried to provide an effective reference for the government network public opinion [17]. Wang Huijun and other people applied the chaotic theory to study the chaos characteristics of the network public opinion event risk. And they discussed the risk evaluation theory of the network public opinion which was based on the chaos theory [18]. In addition, Sun Jianguo, Fang Aigui and other people also studied the network public opinion.

From the above literatures, we can find that the evaluated study for the network public opinion is very rare. It is necessary to evaluate the network public opinion and take appropriate measures to reduce the risk of the network public opinion. In this paper, according to the characteristics of the network public opinion, we propose the combination weight AHP-Entropy method, namely CWAHP-Entropy. And we use the method to evaluate the network public opinion. The structure of this paper is as follows. The first part is the introduction. In this part, we introduce the research status of the network public opinion. In the second part, according to the design principle of the evaluated index, we design the risk evaluation index of the network public opinion. The third part is the CWAHP-Entropy method. In the third part, according to the characteristics of the network public opinion, we propose the new evaluated method. The fourth part is the numerical experiment. We use the new evaluation method to evaluate the risk of the network public opinion. And the last part is the conclusion. 


\section{The Design of the Evaluation Index}

The risk evaluation of the network public opinion refers that people evaluate and control the spreading risk of the network public opinion-especially the negative network public opinion from the signs of the network public opinion appeared to the crisis caused the loss. The risk evaluation of the network public opinion is a process that the network public opinion may cause the risk to evaluate. According to evaluate the network public opinion, we can find the possibility of the network public opinion crisis. And we can take the related measures to judgment the direction and size of the possible crisis. Then, we notify the relevant functional departments to do the adequate preparation to face the crisis. In order to evaluate the risk of the network public opinion reasonably, we need to establish a complete evaluation system. The evaluation system must be objective, scientific and reasonable. It can response the emergency capacity as much as possible under the emergency supply chain coordination mechanism. Therefore, we must follow the following principles.

The first principle is the scientific principle. The structure of the evaluation system and the selection of the indicators must have the sufficient scientific basis. We must adhere to the scientific principle. Then, the obtained information is reliability and objectivity. The evaluated results of the network public opinion risk can be more accurate and reasonable.

The second principle is the purpose principle. The purpose of establishing the evaluation index system is to evaluate the risk of the network public opinion, find timely the sign of the network public opinion risk and be enough prepare to face the crisis. Therefore, the index of the network public opinion risk evaluation must follow the purpose principle. And it is helpful to deal with the evaluated results of the network public opinion crisis.

The third principle is the practical principle. The index of the network public opinion risk evaluation should be simplified. Under the condition of ensuring the objectivity and the comprehensiveness, the index system should be simplified as much as possible.

The fourth principle is the combination between the objective index and the subjective index. The evaluated system is composed by several indexes. And these indexes are interrelated and interact with each other. Therefore, when we establish the evaluation system, we need to combine the objective index with the subjective index to ensure the fairness and authority of the evaluation results.

The fifth principle is the operability principle. After the related departments obtain the data from the evaluation system, the managers can operate the network public opinion events which need to be evaluated. Then, they can get the final evaluation results in order to guide the management work of the network public opinion.

Following the above principle, we establish the evaluation indexes of the network public opinion risk. They are shown in the following table.

Table 1. The Evaluation Indexes of the Network Public Opinion Risk

\begin{tabular}{l|l|r} 
Risk & Social & Educational gap
\end{tabular}




\begin{tabular}{|c|c|c|}
\hline \multirow{11}{*}{$\begin{array}{l}\text { evaluation } \\
\text { index of the } \\
\text { network } \\
\text { public } \\
\text { opinion }\end{array}$} & \multirow[t]{3}{*}{ influence } & Employment rate \\
\hline & & Crime rate \\
\hline & & Income gap \\
\hline & \multirow{4}{*}{$\begin{array}{l}\text { Personal } \\
\text { influence }\end{array}$} & $\begin{array}{c}\text { The attention degree of the negative network public } \\
\text { opinion }\end{array}$ \\
\hline & & The trust degree of the negative network public opinion \\
\hline & & $\begin{array}{l}\text { The proportion of maintaining an optimistic attitude for } \\
\text { the public opinion }\end{array}$ \\
\hline & & The proportion of self -awareness for the public opinion \\
\hline & \multirow{4}{*}{$\begin{array}{l}\text { Government } \\
\text { influence }\end{array}$} & The influence on the judicial justice \\
\hline & & The influence on the government supervision \\
\hline & & $\begin{array}{l}\text { The influence on the transparency of the government } \\
\text { decision making }\end{array}$ \\
\hline & & The influence on the public decision making \\
\hline
\end{tabular}

\section{The CWAHP-Entropy}

\subsection{Entropy Weight Method}

The concept of entropy was firstly proposed by German physicist Clausis and Boltgman. Later, American mathematician, the founder of control theory and information theory, Wiener and Shanon presented broader information entropy. Now, it is widely used in the engineering technology, social economy and other fields. Entropy method is a kind of objective weighting method that using their own information to judgment the effectiveness and the value. It can make the objective and fair evaluation for the system.

The specific calculation steps of the entropy method are as follows.

We assume that there are ${ }^{n}$ evaluation objects in the evaluation system. The index system includes $m$ evaluation indexes. Then we can get an $m \times n$ original data evaluation matrix.

The first step is the data standardization.

In the evaluation system, the dimension of each index, the dimensional unit and the orientation of the index can be different. Therefore, when we evaluate the system, we need to deal with the data standardization. For the positive index, the bigger the index value is, the better. For the negative index, the smaller the index value is the better. The calculation formula for the positive and negative index are (1) and (2).

The positive index:

$\chi_{i j}^{\prime}=\frac{\chi_{i j}-\chi_{j \min }}{\chi_{j \max }-\chi_{j \min }}(i=1,2, \cdots, n ; j=1,2, \cdots, m)$

The negative index:

$\chi_{i j}^{\prime}=\frac{\chi_{j \max }-\chi_{i j}}{\chi_{j \max }-\chi_{j \min }}(i=1,2, \cdots, n ; j=1,2, \cdots, m)$

Where, $\chi_{i j}$ is the $j$ index for the $i$ evaluation object. $\chi_{j \min }$ is the minimum value for the $j$ index. $\chi_{j \max }$ is the maximum value for the $j$ index. Then, we get the data standardization matrix.

$$
X=\left\{\begin{array}{cccc}
\chi_{11}^{\prime} & \chi_{12}^{\prime} & \cdots & \chi_{1 m}^{\prime} \\
\chi_{21}^{\prime} & \chi_{22}^{\prime} & \cdots & \chi_{2 m}^{\prime} \\
\vdots & \vdots & \cdots & \vdots \\
\chi_{n 1}^{\prime} & \chi_{n 2}^{\prime} & \cdots & \chi_{m n}^{\prime}
\end{array}\right\}
$$


In order to avoid the zero data, we need to translate the index data. The formula is shown in (3).

$$
\chi_{i j}^{\prime}=\chi_{i j}^{\prime}+1
$$

Then, we normalize the data matrix and the calculation formula is shown in (4).

$$
p_{i j}=\frac{\chi_{i j}^{\prime}}{\sum_{i=1}^{n} \chi_{i j}^{\prime}}, \quad 0 \leq p_{i j} \leq 1
$$

From the formula, we can obtain the standard matrix ${ }^{P=\left(p_{i j}\right)_{n \times m}}$.

The second step is to calculate the information entropy for the ${ }^{j}$ index.

$$
e_{j}=-k \sum_{i=1}^{n} P_{i j} \cdot \ln P_{i j}
$$

Where, $\quad k=\frac{1}{\ln m}, j=1,2, \cdots, m$. According to ${ }^{k}$, we can find that ${ }^{e_{j}}$ is in ${ }^{[0,1]}$.

The third step is to calculate the difference coefficient for the ${ }^{j}$ index.

$$
g_{j}=1-e_{j}(j=1,2, \cdots, m)
$$

For the fixed ${ }_{j}$ index, the smaller the difference $\chi_{i j}$ is, the bigger the information entropy is. Then, the influence of the index on the scheme is smaller. When the index difference of each scheme is bigger, the information entropy is smaller. Then, the influence of the index on the scheme is bigger.

The fourth step is to calculate the weight for the $j$ index.

$v_{j}=\frac{g_{j}}{\sum_{j=1}^{m} g_{j}}(j=1,2, \cdots, m)$

\subsection{The Improved AHP-Entropy Method Considering Combination Weight}

3.2.1 The Calculation of the Subjective Weight: We use the cluster analysis method to calculate the weight of the experts. The experts adhere to the majority principle. Now, we assume that the weight of the decision expert is $\varepsilon=\left(\varepsilon_{1}, \varepsilon_{2}, \cdots, \varepsilon_{k}\right)$ and $\sum_{i=1}^{k} \varepsilon_{i}=1$. The specific steps are as follows.

Firstly, we assume that each decision expert is a class. And we construct $k$ classes $G=\left(G_{1}, G_{2}, \cdots, G_{k}\right) \cdot g=k$

Secondly, according to formula (8), we calculate the normalized vector of the judgment matrix for each decision expert.

$b^{\prime}=\left(b_{i}^{l}\right)_{n \times 1}=\frac{1}{n} \sum_{j=1}^{n} b_{i j}^{l}=\frac{1}{n}\left(p_{i j}^{l} / \sum_{i=1}^{n} p_{i j}^{l}\right)$

Thirdly, according to the formula (9), we calculate the consistency degree between the two classes.

$$
d_{i j}=\left(b^{i} \cdot b^{j}\right) /\left(\left|b^{i}\right| \cdot\left|b^{j}\right|\right)
$$

Fourthly, we find the maximum ${ }^{d_{x y}}$ in the consistency degree value ${ }^{d_{i j}}$. And we merge the corresponding ${ }^{G_{x}}, G_{y}$ to a new class ${ }^{G_{g+1}}$. That is ${ }^{G_{g+1}}=\left(G_{x}, G_{y}\right)$. 
Fifthly, if $g=2(k-1)$, we turn to the step (9). Otherwise, we turn to the step (6).

Sixthly, in the class set, we remove $G_{x}, G_{y}$ and add $G_{g+1}$.

Seventhly, we calculate the consistency degree value for the new two classes.

$$
d_{i, q+1}=\max \left\{d_{i x}, d_{i y}\right\},(i \neq x, y)
$$

Eighthly, return to the step (3), and we continue to merge the rest classes. $g=g+1$

Ninthly, we determine the number of the classes and the classes.

Lastly, we calculate the weight of the decision expert.

$$
\varepsilon_{l}=\psi_{g} / \sum_{l=1}^{k} \psi_{g}^{l}
$$

Where, $\psi_{g}$ is the number of the experts in the class.

Then, we assume that the weight for the target attributes is,

$$
\theta=\left(\theta_{1}, \theta_{2}, \cdots, \theta_{n}\right) \text { and } \sum_{j=1}^{n} \theta_{j}=1 .
$$

According to the above calculation, we can obtain the individual weight of the decision expert. Then, we apply the weighted logarithm least square method to calculate.

$$
\min f(\theta)=\sum_{i=1}^{n} \sum_{j=1}^{n} \sum_{l=1}^{k} \varepsilon_{l}\left[\ln \left(p_{i j}^{l}\right)-\ln \left(\theta_{j} / \theta_{i}\right)\right]^{2}
$$

We make $\partial f(\theta) / \partial \theta_{j}=0$. Then,

$$
\theta_{j}=\frac{\left[\prod_{i=1}^{n} \prod_{l=1}^{k} p_{j i}^{\varepsilon}\right]^{l / n}}{\sum_{i=1}^{n}\left[\prod_{i=1}^{n} \prod_{l=1}^{k} p_{j i}^{\varepsilon}\right]^{l / n}}
$$

3.2.2 The Combination of the Subjective Weight and the Objective Weight: According to the formula (11), we calculate the subjective and objective weights of the target attributes which are obtained by applying the entropy method and the group AHP method. Then, we get the combined weights $\omega=\left(\omega_{1}, \omega_{2}, \cdots, \omega_{n}\right)$. And we make it as the weight of each attribute. This weight not only considers the objective factors, but also the subjective factors. It can reflect comprehensively the relative importance degree of the attributes.

$\omega_{j}=\alpha \eta_{j}+\beta \theta_{j}$

In the formula, $\alpha$ is the influence factor of the objective weight. $\beta$ is the influence factor of the subjective weight.

$\alpha+\beta=1$

The defined principle is as follows. According to the integrity, the credibility of the airborne sensor data information and the trust degree of the experts, we combine the specific air wars to determine the final subjective and objective influence factors.

\subsection{The Fuzzy Hierarchy Evaluation Method}

We assume that $U=\left\{u_{1}, u_{2}, \cdots, u_{m}\right\}$ is the index factor and $V=\left\{v_{1}, v_{2}, \cdots, v_{n}\right\}$ is the evaluation set. When evaluating $B=\left\{b_{1}, b_{2}, \cdots, b_{m}\right\}$, the fuzzy evaluation method rely on the weight of each factor. It is the fuzzy subset $A=\left\{a_{1}, a_{2}, \cdots, a_{m}\right\}$ in $U$ and $\sum_{i=1}^{m} a_{i}=1 . a_{i}$ is the 
weight of the $i$ factor. It reflects the important degree for each index factor in the fuzzy evaluation method. And it meets the unit and non-negative condition.

The steps of the fuzzy evaluation method are as follows.

Firstly, we determine the factor $\operatorname{set}^{U}=\left\{u_{1}, u_{2}, \cdots, u_{m}\right\}, u_{i}(i=1,2, \cdots, m)$ shows the factor of the evaluation question.

Secondly, we determine the evaluation $\operatorname{set}^{V}=\left\{v_{1}, v_{2}, \cdots, v_{n}\right\}, v_{j}(j=1,2, \cdots, n)$ shows that the ${ }^{j}$ rank of the evaluation question. We can set up the evaluation criteria of the evaluation set according to the qualitative analysis.

Thirdly, we determine the weight of each factor $A=\left(a_{1}, a_{2}, \cdots a_{m}\right) \cdot a_{i}(i=1,2, \cdots, n)$ is the important degree of the factor $u_{i}(i=1,2, \cdots, m)$ for other factors. And it meets $\sum_{i=1}^{m} a_{i}=1$.

Fourthly, we evaluate it. Firstly, the expert evaluates $u_{i}(i=1,2, \cdots, m)$ and determines the grade for $v_{j}(j=1,2, \cdots, n)$. Then, we obtain the bottom evaluation matrix.

$$
R=\left\{\begin{array}{cccc}
r_{11} & r_{12} & \cdots & r_{1 n} \\
r_{21} & r_{22} & \cdots & r_{2 n} \\
\vdots & \vdots & \vdots & \vdots \\
r_{m 1} & r_{m 2} & \cdots & r_{m n}
\end{array}\right]
$$

Where, $R$ is a fuzzy relation from the factor set $U=\left\{u_{1}, u_{2}, \cdots, u_{m}\right\}$ to the evaluation set $V=\left\{v_{1}, v_{2}, \cdots, v_{n}\right\}, r_{i j}$ is the membership degree of the evaluation grade $v_{j}$ for the factor $u_{i}$. And it meets $\sum_{j=1}^{n} r_{i j}=1$.

Then, we transfer a fuzzy set of the factor $\operatorname{set}^{U}=\left\{u_{1}, u_{2}, \cdots, u_{m}\right\}$ to a fuzzy set of the comment set $V=\left\{v_{1}, v_{2}, \cdots, v_{n}\right\}$ according to the fuzzy relation $R$. When the weight and the evaluation matrix are known, we can evaluate according to the following transformation.

$$
\begin{aligned}
B & =A \circ R=\left(a_{1}, a_{2}, \cdots a_{m}\right){ }^{\circ} R \\
& =\left(a_{1}, a_{2}, \cdots a_{m}\right) \circ\left[\begin{array}{cccc}
r_{11} & r_{12} & \cdots & r_{1 n} \\
r_{21} & r_{22} & \cdots & r_{2 n} \\
\vdots & \vdots & \vdots & \vdots \\
r_{m 1} & r_{m 2} & \cdots & r_{m n}
\end{array}\right] \\
& =\left(b_{1}, b_{2}, \cdots, b_{n}\right)
\end{aligned}
$$

$B$ is a fuzzy subset in the comment set $V=\left\{v_{1}, v_{2}, \cdots, v_{n}\right\} . \circ$ is the fuzzy operator. $\left(b_{1}, b_{2}, \cdots, b_{n}\right)$ is the membership degree of the fuzzy set and it meets $\sum_{j=1}^{n} b_{j}=1$.

Then, we do the fuzzy evaluation for the two level indexes. The two level evaluations are to evaluate the lowest level evaluated index. Therefore, the evaluation matrix is as follows.

$$
R^{2}=\left[\begin{array}{c}
B_{1} \\
B_{2} \\
\vdots \\
B_{n}
\end{array}\right]=\left[\begin{array}{cccc}
b_{11} & b_{12} & \cdots & b_{1 p} \\
b_{21} & b_{22} & \cdots & b_{2 p} \\
\vdots & \vdots & \vdots & \vdots \\
b_{n 1} & b_{n 2} & \cdots & b_{n p}
\end{array}\right]
$$

The weight of the factor $\operatorname{set} U=\left\{u_{1}, u_{2}, \cdots, u_{m}\right\}$ is $A=\left(a_{1}, a_{2}, \cdots a_{m}\right)$. According to the first layer fuzzy evaluation method, we can obtain the two level fuzzy evaluations. 


$$
\begin{aligned}
& B=A^{\circ} R^{2}=\left(a_{1}, a_{2}, \cdots a_{m}\right)^{\circ} R^{2} \\
& =\left(a_{1}, a_{2}, \cdots a_{m}\right) \circ\left[\begin{array}{cccc}
b_{11} & b_{12} & \cdots & b_{1 p} \\
b_{21} & b_{22} & \cdots & b_{2 p} \\
\vdots & \vdots & \vdots & \vdots \\
b_{n 1} & b_{n 2} & \cdots & b_{n p}
\end{array}\right] \\
& =\left(b_{1}, b_{2}, \cdots, b_{p}\right)
\end{aligned}
$$

And so on, we can get the final evaluation results.

\section{Numerical Experiment}

We apply the method that this paper proposes to evaluate the risk of the network public opinion. Firstly, we need to determine the subjective weight of the target attribute. We make the social influence in the index system as the example. In the social influence factor, we invite three experts to evaluate. And we calculate the normalized vector according to the judgment matrix.

$$
\begin{aligned}
& b_{1}=(0.09653,0.27583,0.46857 .0 .15907) \\
& b_{2}=(0.12034,0.25670,0.43896 .0 .18400) \\
& b_{3}=(0.11542,0.46592,0.30573 .0 .11292)
\end{aligned}
$$

Then, we get the consistency degree of the decision experts.

$$
\begin{aligned}
& d_{12}=0.9874 \\
& d_{13}=0.8654 \\
& d_{23}=0.8962
\end{aligned}
$$

Therefore, the first decision expert and the second decision expert compose the new class. And the third decision expert is another new class. In the new class, the number of the experts is two in the first class. In the second class, the number of the expert is one.

Then, we can get

$$
\begin{gathered}
\varepsilon_{1}=\varepsilon_{2}=2 /(2+2+1)=0.4 \\
\varepsilon_{3}=1 /(2+2+1)=0.2
\end{gathered}
$$

The subjective weight of the target attribute is,

$$
w_{1}^{1}=(0.13652,0.29878,0.36523,0.19947)
$$

Then, we apply the entropy method to calculate the objective weight of the target.

$$
w_{2}^{1}=(0.15620,0.27781,0.34412,0.22187)
$$

Therefore, we can get the weights of the four indexes about the social influence.

$$
w^{1}=0.5 w_{1}^{1}+0.5 w_{2}^{1}=(0.1464,0.2883,0.3547,0.2107)
$$

According to this method, we can get the index weight of the internet public opinion. It is shown in the table 2 .

Table 2. The Weights of each Indexes

\begin{tabular}{|c|c|c|c|}
\hline Second index & Weight & Three index & Weight \\
\hline \multirow{3}{*}{ Social influence } & \multirow{3}{*}{0.3347} & Education gap & 0.1464 \\
\cline { 3 - 4 } & & Employment rate & 0.2883 \\
\cline { 3 - 4 } & & Crime rate & 0.3574 \\
\cline { 3 - 4 } & 0.2567 & The attention degree of the negative network & 0.2107 \\
\hline Personal & 0.2874 \\
\hline
\end{tabular}




\begin{tabular}{|c|c|c|c|}
\hline \multirow{3}{*}{ influence } & \multicolumn{1}{|c|}{ public opinion } & \\
\cline { 3 - 4 } & & $\begin{array}{c}\text { The trust degree of the negative network public } \\
\text { opinion }\end{array}$ & 0.3641 \\
\cline { 3 - 4 } & & $\begin{array}{c}\text { The proportion of maintaining an optimistic } \\
\text { attitude for the public opinion }\end{array}$ & 0.2097 \\
\cline { 3 - 4 } & & $\begin{array}{c}\text { The proportion of self -awareness for the public } \\
\text { opinion }\end{array}$ & 0.2388 \\
\hline \multirow{3}{*}{$\begin{array}{c}\text { Government } \\
\text { influence }\end{array}$} & \multirow{4}{*}{0.4086} & The influence on the judicial justice & 0.3513 \\
\hline & & $\begin{array}{c}\text { The influence on the government supervision } \\
\text { The influence on the transparency of the } \\
\text { government decision making }\end{array}$ & 0.2369 \\
\hline & & The influence on the public decision making & 0.1462 \\
\hline
\end{tabular}

After getting the weight, we apply the fuzzy hierarchy evaluation method to evaluate the risk of the network public opinion.

We also take the social influence as an example and get the evaluation value.

\section{Table 3. The Score of the Indexes}

\begin{tabular}{|c|c|c|}
\hline Index & Evaluation grade & Evaluation value \\
\hline \multirow{4}{*}{ Education gap } & poor & 0 \\
\cline { 2 - 3 } & bad & 0.4 \\
\cline { 2 - 3 } & normal & 0.4 \\
\cline { 2 - 3 } & good & 0.2 \\
\hline \multirow{5}{*}{ Employment rate } & excellent & 0 \\
\cline { 2 - 3 } & poor & 0 \\
\cline { 2 - 3 } & bad & 0.1 \\
\cline { 2 - 3 } & normal & 0.5 \\
\cline { 2 - 3 } & good & 0.2 \\
\hline \multirow{5}{*}{ Crime rate } & excellent & 0.2 \\
\cline { 2 - 3 } & poor & 0 \\
\cline { 2 - 3 } & bad & 0.2 \\
\cline { 2 - 3 } & normal & 0.5 \\
\cline { 2 - 3 } & good & 0.2 \\
\hline \multirow{5}{*}{ Income gap } & excellent & 0.1 \\
\cline { 2 - 3 } & poor & 0 \\
\cline { 2 - 3 } & bad & 0.1 \\
\cline { 2 - 3 } & normal & 0.4 \\
\cline { 2 - 3 } & good & 0.3 \\
\hline \multirow{5}{*}{} & excellent & 0.2 \\
\hline
\end{tabular}

$$
B_{1}=w^{1} \cdot R^{1}=(0.1464,0.2883,0.3574,0.2107) \cdot\left[\begin{array}{ccccc}
0 & 0 & 0.4 & 0.4 & 0.2 \\
0 & 0.1 & 0.5 & 0.2 & 0.2 \\
0 & 0.2 & 0.5 & 0.2 & 0.1 \\
0 & 0.1 & 0.4 & 0.3 & 0.2
\end{array}\right]
$$

$=(0,0.1214,0.4657,0.2509,0.1648)$

In the same way, we can get 


$$
\begin{aligned}
& B_{2}=w^{2} \cdot R^{2}=(0.2874,0.3641,0.2097,0.2388) \cdot\left[\begin{array}{lllll}
0 & 0.2 & 0.4 & 0.3 & 0.1 \\
0 & 0.1 & 0.3 & 0.3 & 0.3 \\
0 & 0.2 & 0.3 & 0.2 & 0.3
\end{array} \mid\right. \\
& =(0,0.1597,0.3587,0.3329,0.2486) \\
& B_{3}=w^{3} \cdot R^{3}=(0.3513,0.2369,0.1462,0.2656) \cdot\left[\begin{array}{lllll}
0 & 0.2 & 0.4 & 0.3 & 0.1 \\
0 & 0.2 & 0.3 & 0.4 & 0.1 \\
0 & 0.2 & 0.5 & 0.1 & 0.2 \\
0 & 0.1 & 0.4 & 0.4 & 0.1
\end{array}\right] \\
& =(0,0.1734,0.3909,0.3210,0.1146) \\
& S=w \cdot R=(0.3347,0.2567,0.4086) \cdot\left[\begin{array}{lllll}
0 & 0.1214 & 0.4657 & 0.2509 & 0.1648 \\
0 & 0.1597 & 0.3587 & 0.3329 & 0.2486 \\
0 & 0.1734 & 0.3909 & 0.3210 & 0.1146
\end{array}\right] \\
& =(0,0.1525,0.4077,0.3006,0.1658)
\end{aligned}
$$

The comment set is

$$
\begin{aligned}
V & =(\text { poor }, \text { bad }, \text { normal }, \text { good }, \text { excellent }) \\
& =(50,60,70,80,90)
\end{aligned}
$$

The evaluation result is $F=S \cdot V=76,659$.

\section{Conclusion}

At present, more and more influent network public opinion are forming gradually. With the increase of the number of the Internet users, the network public opinion and the reality of the social public opinion converge gradually. The network public opinion has become a microcosm of the social media. China is in the period of the social transformation. There are some inevitable social contradictions. These contradictions may be used by some unscrupulous elements. And it results the serious social influence. Therefore, it is necessary to evaluate the risk of the network public opinion. The major contents of this paper are as follows. Firstly, this paper introduces the research status at home and domestic. Secondly, this paper designs the risk evaluation index of the network public opinion according to the principle of the evaluation index. Thirdly, this paper proposes the CWAHP-Entropy method. Finally, this paper uses the new evaluation method to evaluate the risk of the network public opinion. The experimental results show that the method ha the reliability and the validity.

\section{References}

[1] L Bogao. "Public opinion administration of government [M]".Beijing, The communication university of China press, (2008).

[2] L Jianming. "Principle of public opinion [M]". Bei Jing, Huaxia Press, (2002).

[3] T Shoulian. "Networking and Its Governance [M]". Bei Jing, Beijing University of Posts and Telecommunications press (2008).

[4] W Tianyi. "Network opinion guide and the construction of harmonious Forum [M]". Bei Jing, People's press, (2008).

[5] Y Guoming. "Annual report of public opinion in China [M]". Bei Jing, People's daily press, (2014).

[6] J Urban, K Bulkow. "Tracing Public Opinion Online - An Example of Use for Social Network Analysis in Communication Research [J]”. Procedia-Social and Behavioral Sciences, vol. 100, no. 7, (2013), pp. 108-126.

[7] N Ma, Y Liu. "peredgeRank algorithm and its application in identifying opinion leader of online public opinion supernetwork [J]", . Expert Systems with Applications, vol. 41, no. 4, (2014), pp.1357-1368.

[8] C G. Reddick, A Takeoka Chatfield, P A. Jaramillo. "Public opinion on National Security Agency surveillance programs: A multi-method approach [J]". Government Information Quarterly, vol. 32, no. 2, (2015), pp. 129-141. 
[9] P Porten-Cheé, C Eilders. "Spiral of silence online: How online communication affects opinion climate perception and opinion expression regarding the climate change debate [J]". Studies in Communication Sciences, In Press, Corrected Proof, Available online 29 March (2015).

[10] K Kim, Y Min Baek, N Kim. "Online news diffusion dynamics and public opinion formation: A case study of the controversy over judges' personal opinion expression on SNS in Korea [J]". The Social Science Journal, vol. 52, no. 2, (2015), pp. 205-216.

[11] N Ma, Y Liu. "SuperedgeRank algorithm and its application in identifying opinion leader of online public opinion supernetwork [J]". Expert Systems with Applications, vol. 41, no. 4, (2014), pp. 1357-1368.

[12] O Bayulgen, E Arbatli. "Cold War redux in US-Russia relations? The effects of US media framing and public opinion of the 2008 Russia-Georgia war [J]". Communist and Post-Communist Studies, no. 46, (2013), pp. 513-527.

[13] L.M. Stapleton, P. Hanna, N. Ravenscroft, “A. Church. Flexible ecosystem services proto-typology based on public opinion [J]”. Ecological Economics, no. 106, (2014), pp. 83-90.

[14] Y Yuanyuan, "The emergence and characteristics of Internet public opinion [D]". Hunan Normal University, Journalism, (2007).

[15] L Hao, "The research on the formation mechanism and control strategy of network public opinion [D]". University of Electronic Science and technology, Communication Science, (2004).

[16] Z Wei, "The research on the influence of network public opinion and its control [D]". Guang Xi University, Journalism, (2008).

[17] Z Ying, Y Xiucai. "An analysis of the public opinion events and its characteristics from 1998 to 2009 [J]". Journalism and Communication Studies, no. 4, (2010), pp. 45-52.

[18] W Huijun. "Risk assessment of network public opinion events based on Chaos Theory [J]". Enterprise economy, no. 2, pp. 74-76.

[19] S Jianguo, "Early warning and security of the Internet Opinion [D]", Chongqing University, Journalism, (2010).

[20] F Aigui, "The research of the impact of Internet public opinion on public policy [D]", North China Electric Power University, Administration, (2013). 
International Journal of Security and Its Applications

Vol. 10, No. 4 (2016) 